\title{
Far Ultraviolet Observations of Hot DA White Dwarfs
}

\author{
David Finley, Gibor Basri, ${ }^{\dagger}$ and Stuart Bowyer ${ }^{\ddagger}$ \\ Space Sciences Laboratory, University of California, Berkeley \\ $\dagger$ Astronomy Department, University of California, Berkeley \\ $¥$ Astronomy Department and Space Sciences Laboratory, \\ University of California, Berkeley
}

\begin{abstract}
Far ultraviolet (FUV) fluxes have been used for determining the effective temperatures of a number of DA white dwarfs hotter than $20,000 \mathrm{~K}$. The spectra were obtained with the International Ultraviolet Explorer (IUE). The analysis consisted of comparing the observed FUV fluxes with model fluxes scaled to the V-band flux. After suitable corrections were performed for the time-dependent sensitivity degradation of the IUE, it was found that the available flux calibrations for the IUE were insufficiently accurate for precise temperature determinations. Accordingly, we used seven white dwarfs for which accurate, independent temperature determinations have been made from line profile analyses to improve the accuracy of the IUE flux calibration. The correction to the original calibration was as great as $20 \%$ in individual $5-\AA$ wavelength bins, while the average over the IUE wavelength range was $5 \%$. We present both our IUE flux correction and the temperatures obtained for the hot white dwarfs.
\end{abstract}

\section{Introduction}

Accurate temperature determinations for hot DA white dwarfs are necessary for several reasons. Temperatures are needed for deriving the luminosity function of DA's; the luminosity function then serves to check calculations of cooling rates for these stars. Trace element abundances in DA's result from temperaturedependent processes. Successful confrontation of observational abundance determinations with theory requires that the effective temperatures be known with sufficient accuracy. Also, the upper temperature limit for DA's needs to be determined with reasonable precision, because this limit will help constrain post main sequence evolutionary calculations. Additionally, upcoming extreme ultraviolet (EUV) photometric survey missions (Bowyer 1983, Pye and Page 1987) are likely to discover hundreds of very hot DA white dwarfs (Finley 1988). Non-EUV measurements will be required to make the accurate temperature determinations necessary for interpretation of the EUV photometric data for these stars (Finley 1988).

Three basic types of measurements are available for determining temperatures of hot white dwarfs: optical continua (from photometry), FUV continua, and line profiles. Optical photometry is insufficiently accurate for the hotter $(>25,000 \mathrm{~K}$ ) white dwarfs. Line profiles and FUV continua give results of comparable accuracy. We present here our temperature determinations based on FUV continuum measurements.

\section{Observations and Data Reduction}

We conducted a program of observations of hot DA white dwarfs with the International Ultraviolet Explorer satellite (IUE). A description of the IUE instrumentation is presented in Boggess et al. (1978a,b). Our observations consisted primarily of low dispersion exposures using the SWP and LWR cameras. Additionally, a number of spectra obtained from the IUE data archives are included in the results presented here. In processing the data, the SWP spectra were truncated shortward of $1320 \AA$ to avoid the wing of the $L \alpha$ line. An extended wavelength baseline was obtained by including the V-band flux, which was calculated per the relation $f(5490 \AA)=3.61 \times 10^{-9} / 10^{0.4 m_{\mathrm{v}}} \mathrm{erg} / \mathrm{cm}^{2} \sec \AA$.

\section{Data Analysis Technique}

The IUE data were analyzed by comparing observed fluxes with model fluxes that were calculated with Basri's white dwarf model atmosphere code (Malina, Bowyer, and Basri 1982). Based on Auer's complete linearization method, this is an LTE, hydrogen line-blanketed atmosphere code, which omits convective energy transport. The atmosphere code was used to generate a grid of models at different effective temperatures between $20,000 \mathrm{~K}$ and $100,000 \mathrm{~K}$, while $\log g$ and $\mathrm{n}(\mathrm{He}) / \mathrm{n}(\mathrm{H})$ were held fixed at 8 and $1 \times 10^{-6}$. The 
variation of $\log g$ within the white dwarf range was found to have an insignificant effect on the FUV flux. Inferred temperatures based on FUV fluxes vary by only $\pm 3 \mathrm{~K}$ at $30,000 \mathrm{~K}$ if $\log g$ is allowed to vary from 7 to 9 , for the case of $n(\mathrm{He}) / \mathrm{n}(\mathrm{H})=1 \times 10^{-6}$. Over the allowable range of $n(\mathrm{He}) / \mathrm{n}(\mathrm{H})$ for DA white dwarfs, 0 to $\leq 10^{-2}$, the variation of the helium fraction has a relatively small effect on the continuum flux. Variations in this range change the average FUV flux (relative to the visible) by at most $\sim 3 \%$. Furthermore, it is not possible to solve separately for both $T_{\text {eff }}$ and $n(H e) / n(H)$, because variations in $n(H e) / n(H)$ produce effects on the continuum flux which are indistinguishable from the effects of temperature variations. The shape of the continuum does vary with $n(\mathrm{He}) / \mathrm{n}(\mathrm{H})$, but only at the level of $\leq 0.1 \%$. Consequently, there was no advantage to performing the analysis with $n(\mathrm{He}) / \mathrm{n}(\mathrm{H})$ as a free parameter.

The effective temperatures were obtained by comparing the stellar FUV/visible flux ratios to the model FUV/visible flux ratios. The calculated stellar-to-model ratio at each wavelength is defined as

$$
\delta\left(\lambda_{j}\right)=-2.5 \log \left[\frac{f\left(\lambda_{j}\right) / f\left(\lambda_{\mathrm{V}}\right)}{\mathrm{H}\left(\mathrm{T}_{\mathrm{eff}}, \lambda_{j}\right) / \mathrm{H}\left(\mathrm{T}_{\mathrm{eff}}, \lambda_{\mathrm{V}}\right)}\right],
$$

where $f$ is the measured stellar flux, while $\mathbf{H}$ is the Eddington flux. The $\lambda_{j}$ are the IUE data wavelength points, and $\lambda_{\mathrm{V}}=5490 \AA$. The stellar effective temperatures were found by calculating the model effective temperatures which gave

$$
S=\Sigma \delta\left(\lambda_{j}\right)=0
$$

\section{Temperature Determinations}

The nominal IUE fluxes were corrected for the time-dependent sensitivity variations of the IUE cameras per the prescription of Bohlin (Bohlin and Grillmair 1988a,b), giving fluxes corresponding to the "May 1980" IUE flux calibration (Bohlin and Holm 1980). If desired, the fluxes may be transformed to the 1986 IUE calibration (Bohlin 1986) or the proposed 1988 IUE calibration (Bohlin 1988). However, none of those calibrations give fluxes for white dwarfs which are in reasonable agreement with model atmosphere predictions based on effective temperatures determined from hydrogen line profile measurements. At $60,000 \mathrm{~K}$, for example, the 1980 calibration would give an inferred temperature of only 52,500 K based on the IUE continuum flux. Correspondingly, the 1986 and 1988 calibrations would give temperatures of 53,500 K and 46,900 K, respectively. These inferred temperatures are inconsistent with the line profile temperatures at the 2 to $4 \sigma$ level.

The inconsistency between line profile and FUV continuum flux temperatures was removed thus: we used spectra from seven hot white dwarfs for which accurate temperatures were available to derive flux corrections for the IUE calibration. The temperatures for those stars, based on IUE $L \alpha$ profiles, were obtained by Holberg, Wesemael, and Basile (1986, hereafter referred to as HWB). Given their quoted uncertainties, these are the most accurate hot DA white dwarf temperature measurements presented to date. The errors quoted by HWB are $\pm 3,500 \mathrm{~K}$ at $60,000 \mathrm{~K} ; \pm 1,500 \mathrm{~K}$ at $40,000 \mathrm{~K}$; and $\pm 300 \mathrm{~K}$ at $20,000 \mathrm{~K}$.

Time-corrected fluxes (SWP + LWR) for WD0050-332, 0501+527, 0549+158, 1254+223, 1620-391, $2111+498$, and $2309+105$ were used to obtain the flux correction. For each star, a model was calculated at the temperature presented by HWB. Next, the $R_{i}\left(\lambda_{j}\right)$ values were calculated for the individual stars per

$$
R_{i}\left(\lambda_{j}\right)=\frac{f\left(\lambda_{j}\right) / f\left(\lambda_{\mathrm{V}}\right)}{\mathrm{H}\left(\mathrm{T}_{\mathrm{eff}}, \lambda_{j}\right) / \mathrm{H}\left(\mathrm{T}_{\mathrm{eff}}, \lambda_{\mathrm{V}}\right)} .
$$

Then the flux correction was calculated per

$$
\operatorname{COR}\left(\lambda_{j}\right)=\sum_{i}\left[R_{i}\left(\lambda_{j}\right) I D_{i}\left(\lambda_{j}\right)\right] / \sum_{i} I D_{i}\left(\lambda_{j}\right)
$$

$I D_{i}$ is the data quality flag for each spectrum ( $1 \equiv$ good data, $0 \equiv$ bad data).

The resulting flux correction to the 1980 IUE photometric calibration, binned over $5-\AA$ intervals, is plotted in Figure 1. The correction factor at each wavelength is the value by which a nominal flux must be divided to produce the correct flux. The 1980 IUE flux calibration (relative to the predicted white dwarf fluxes) is seen to give fluxes as much as $15 \%$ high and as much as $20 \%$ low. Averaged over wavelength, the IUE fluxes per the 1980 calibration are low by $5 \%$, which is within the stated overall accuracy for the IUE flux calibration of $\pm 10 \%$. The uncertainty in the correction is dominated by the uncertainties in the V magnitude and line profile temperature determinations for the stars used to calculate the correction. The resulting overall uncertainty in the flux correction, averaged over the IUE wavelength range, was $1.3 \%$.

After applying the flux corrections to the spectra, the effective temperatures were then calculated for all the stars. The temperatures derived from the IUE fluxes are presented in Table 1, along with the temperatures given in HWB, and the temperatures which we have calculated from optical colors. The optical photometric temperatures were based on the multichannel (u-v) color, or on Johnson (U-V) or Stromgren (u-y) colors 


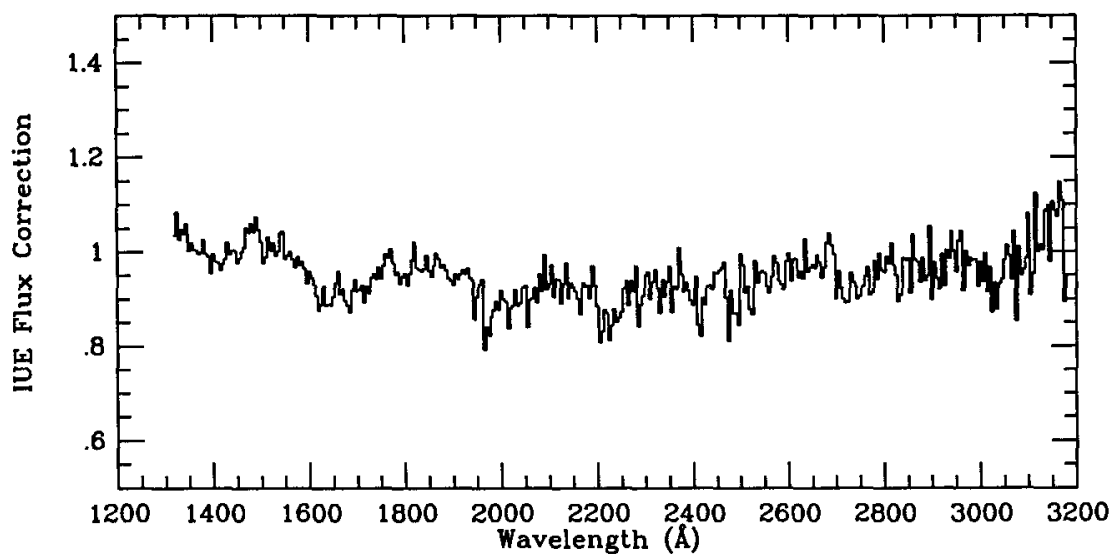

Figure 1. Correction to IUE initial epoch calibration. The correction is based on seven observations of different DA white dwarfs, and is binned into $5 \AA$ intervals. The spectra used were from WD 0050-332, $0501+527,0549+158,1254+223,1620-391,2111+498$, and $2309+105$ (1982/142 observation).

temperatures were based on the multichannel (u-v) color, or on Johnson (U-V) or Stromgren (u-y) colors which were transformed to the equivalent $(u-v)_{M C}$ value. It was necessary to adjust the $(u-v)_{M C}$ or $(u-v)_{M^{-}}$ equivalent colors blueward by 0 m044 to achieve consistency with the line profile and FUV continuum temperatures.

Table 1: Temperatures of stars derived from different measures.

\begin{tabular}{|c|c|c|c|c|c|c|c|}
\hline \multirow[t]{2}{*}{ WD Name } & \multicolumn{2}{|c|}{ IUE Continua } & \multicolumn{3}{|c|}{ Line Profiles } & \multicolumn{2}{|c|}{$\begin{array}{c}\text { Optical } \\
\text { Photometry }\end{array}$} \\
\hline & $\mathrm{T}_{\mathrm{eff}}{ }^{a}$ & Error $^{b}$ & $\mathrm{~T}_{\text {eff }}$ & Error & Ref. & $T_{\text {eff }}$ & Error \\
\hline $0004+330(\mathrm{GD} 2)$ & 46.33 & $\begin{array}{l}+3.44 \\
3.94\end{array}$ & - & -- & $\overline{--}$ & 54.8 & $\begin{array}{ll}+18.2 \\
-100\end{array}$ \\
\hline $0050-432$ (GD659) & 34.15 & +1.64 & 36.85 & \pm 1.39 & HWB & 33.21 & +1.49 \\
\hline 0346-011 (GD50) & 39.10 & $\begin{array}{l}+2.21 \\
-1.90\end{array}$ & 47.5 & \pm 2.5 & $\mathrm{~K}$ & $\begin{array}{l}39.66 \\
31.23\end{array}$ & $\begin{array}{l}+6.54 \\
-4.07 \\
+1.96\end{array}$ \\
\hline $0501+527(\mathrm{G} 191-\mathrm{B} 2 \mathrm{~B})$ & 66.37 & $\begin{array}{l}+8.76 \\
-7.13\end{array}$ & 62.25 & \pm 3.52 & $\mathrm{HWB}$ & 68.7 & $\begin{array}{l}+15.3 \\
10.4\end{array}$ \\
\hline $0549+158(\mathrm{GD} 71)$ & 34.03 & $\begin{array}{c}+1.62 \\
-1.35\end{array}$ & 33.30 & \pm 0.83 & HWB & 37.26 & 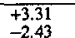 \\
\hline $0644+375$ (EG50) & 21.76 & +0.29 & -- & -. & -- & 22.61 & +6.34 \\
\hline $0651-020$ (GD80) & 35.50 & $\begin{array}{ll}+2.47 \\
-1.96\end{array}$ & -- & $\cdots$ & -- & 36.27 & $\begin{array}{l}+4.48 \\
-2.90\end{array}$ \\
\hline $1031-114(\mathrm{EG} 70)$ & 25.67 & $\begin{array}{l}+0.36 \\
-0.34\end{array}$ & -- & - & -- & 24.91 & $\begin{array}{l}0.49 \\
-0.48\end{array}$ \\
\hline $1033+464(\mathrm{GD} 123)$ & 28.38 & $\begin{array}{l}+4.81 \\
-2.30\end{array}$ & -- & - & -- & $27.2 "$ & \\
\hline $1254+223$ (GD153) & 40.67 & $\begin{array}{l}+2.65 \\
-2.22\end{array}$ & $\begin{array}{l}42.375 \\
42\end{array}$ & $\begin{array}{l} \pm 1.48 \\
\pm 2 \\
\end{array}$ & $\begin{array}{l}\text { HWB } \\
\mathrm{K}\end{array}$ & 41.14 & $\begin{array}{l}+3.23 \\
-2.59\end{array}$ \\
\hline $1403-077(\mathrm{PG})$ & 45.98 & $\begin{array}{l}+8.18 \\
-5.78\end{array}$ & -- & - & -- & 40.89 & ${ }_{-4.53}^{+7.28}$ \\
\hline 1615-154 (EG118). & 31.76 & +0.85 & - & -- & -- & 30.63 & ${ }_{-0.58}^{+0.65}$ \\
\hline $1620-391\left(\mathrm{CD} \cdot 38^{\circ} 10980\right)$ & 24.83 & $\begin{array}{l}+0.39 \\
-0.39\end{array}$ & 24.50 & \pm 0.14 & HWB & 24.26 & ${ }_{-0.46}^{+0.47}$ \\
\hline $2111+498$ (GD394) & 37.36 & $\begin{array}{l}+2.32 \\
-1.91 \\
\end{array}$ & 36.125 & \pm 0.94 & HWB & 36.66 & $\begin{array}{l}+2.43 \\
-1.91\end{array}$ \\
\hline $2309+105(\mathrm{GD} 246)$ & $\begin{array}{l}50.30^{c} \\
52.69^{d}\end{array}$ & $\begin{array}{l}+4.25 \\
-3.57 \\
+4.47 \\
-3.98 \\
\end{array}$ & 53.60 & \pm 2.94 & HWB & 62.8 & $\begin{array}{l}+9.3 \\
-7.1\end{array}$ \\
\hline
\end{tabular}

Temperatures are given in $10^{3} \mathrm{~K}$. ${ }^{b}$ Errors are $1 \sigma$ uncertainties. ${ }^{C}$ Based on 1982142 observations. ${ }^{d}$ Based on $1979 / 355$ observations. References: HWB = Holberg, Wesemael, and Basile (1986). $\mathrm{K}=$ Kahn et al. 1984. 
The line profile and FUV continuum temperatures are consistent for all the objects analyzed, with the exception of WD0346-011, as can be seen in Figure 2a. The optical photometric and IUE temperatures are also consistent, as shown in Figure $2 \mathrm{~b}$, except for WD0346-011. (The lower of the two optical temperatures is based on Greenstein's photometry [Greenstein 1974], which may be more accurate than the measurements which give the higher optical temperature [Kondo et al. 1982]. The WD photometry of Kondo et al. appears to have a somewhat higher dispersion than is generally seen in WD work). The absence of systematic trends in the correlation between the FUV continuum temperatures and the other measurements confirms that any possible errors in the models which affect the continuum differently from the lines are not present at more than the $\sim 1 \%$ level.
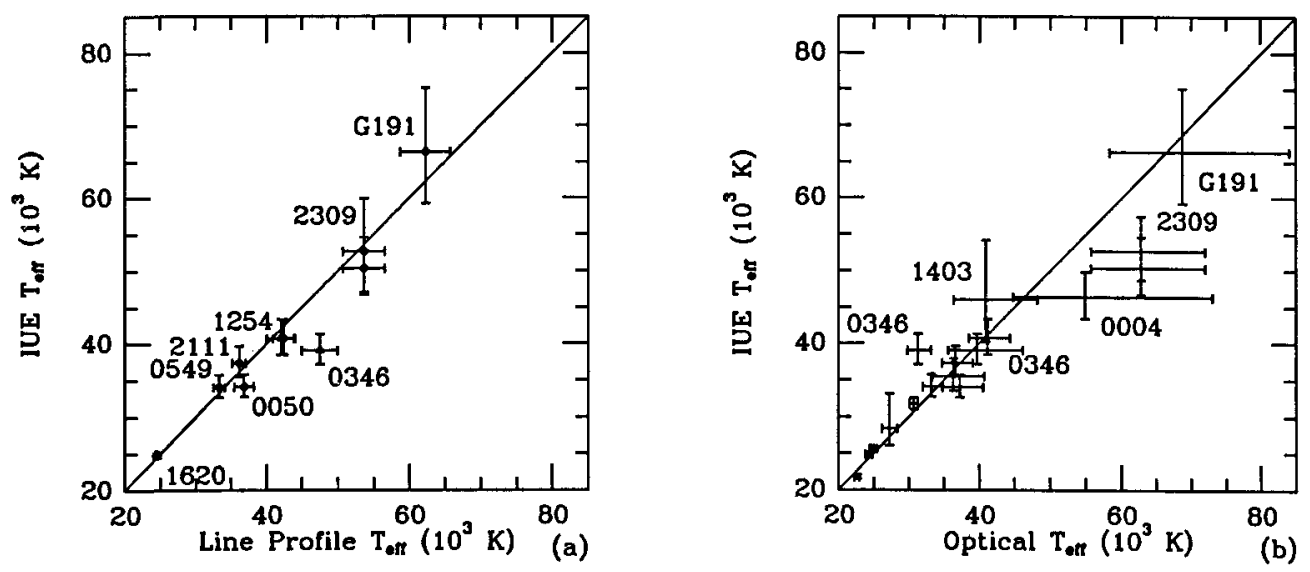

Figure 2. (a) Temperatures derived from IUE fluxes vs. published temperatures calculated from line profile fitting of $H \beta$ (open triangles) or $L \alpha$ (open squares).

(b) Temperatures derived from IUE fluxes $v s$. temperatures based on optical photometry.

\section{Results}

G191-B2B. At $66,400 \mathrm{~K}(+8,800 /-7,100), \mathrm{G} 191-\mathrm{B} 2 \mathrm{~B}$ is among the hottest known DA white dwarfs. It has a well-determined parallax of $0.021 \pm 0.002$ (Routly 1972). This places it at a distance of $47.6 \mathrm{pc} \pm 5 \mathrm{pc}$. Taking $\mathrm{T}_{\text {eff }}=66,380 \mathrm{~K}$ and $\mathrm{m}_{\mathrm{V}}=11.79$, we have $f_{\mathrm{V}} / \mathrm{H}_{\mathrm{V}}=8.58 \times 10^{-22}$. The derived radius is then $0.0170 R_{\odot}$. This value is the same as that predicted by the evolutionary models of Koester and Schönberner (1986) for a $0.6 M_{\odot}$ white dwarf with a hydrogen envelope of $10^{-4} M_{\odot}$. Therefore, G191-B2B may provide an example of a DA white dwarf with a relatively massive remnant hydrogen envelope.

This possible large hydrogen envelope mass would rule out the interpretation offered by Vennes et al. (1987) for the EXOSAT photometric observations of DA white dwarfs. The EXOSAT data indicate that the short wavelength flux is strongly cut off by some absorber within the stellar atmosphere. Vennes et al. suggest that the observed EUV fluxes may result from very thin hydrogen layers overlying the helium. Under their interpretation, the observational results for G191-B2B could be satisfied if the mass of the hydrogen envelope were $\sim 10^{-14.6}$ of the stellar mass, which is inconsistent with the observed radius and the evolutionary models.

WD0346-011. The inconsistent temperatures for this star may be due to the presence of a faint companion; the presence of such a companion is consistent with the optical colors. However, the apparent temperature discrepancies may occur because the star is not a normal DA. WD0346-011 may be a member of a class of white dwarfs currently comprising one object: WD1302+597 (GD323). GD323 has Balmer line profiles consistent with a $\mathrm{T}_{\text {eff }}$ of $40,000 \mathrm{~K}$, but the optical and FUV continua are better fit with a $\mathrm{T}_{\text {eff }}$ of only $30,000 \mathrm{~K}$ (Liebert et al. 1984). Liebert, Fontaine, and Wesemael (1987) point out that the predominantly helium channel of the white dwarf sequence is totally depopulated between $30,000 \mathrm{~K}$ and $45,000 \mathrm{~K}$, and they suggest that GD323 is in transition between DA and DB at the cool end of the helium gap. Perhaps WD0346-011 is undergoing a similar transition at the hot end of the gap.

WD1033+464. This star is composite; hence the optical temperature included in Table 1 is only nominal. The companion, which contributes $\leq 15 \%$ of the light at $5490 \AA$, is probably an $M$ dwarf. The contribution of the companion to the combined light of the system is $\leq 1 \%$ at $3571 \AA$. Therefore, the fiux at that wavelength was used as the visible flux point for the IUE temperature analysis. 
Non-DA Objects. Two of the stars in our observing program are currently classified as DA but were shown on the basis of the IUE spectra to belong to other spectral classes. The two stars in question are WD (or PG) $1247+553$ (GD319), and WD 1544+008 (EG113, BD+01 3129B). 1247+553 is evidently an sdB, while 1544+008 appears to be a helium-rich subdwarf. Of the stars whose spectra were obtained from the archive, 0109-264 (GD691) is also an sdB, while 0823+316 appears to be a very hot hydrogen-poor object. (Analysis of $0823+316$ using pure $H$ models yields $T_{\text {eff }}>100,000 \mathrm{~K}$.)

\section{Conclusions}

Line profile measurements and IUE FUV fluxes (when the latter are suitably corrected) provide very accurate temperatures for hot DA white dwarfs. In most instances, the FUV continuum measurements are equivalent to line profile measurements for the purpose of temperature determinations. When the optical photometry is suitably adjusted, the optical photometric temperatures are consistent with the corrected IUE temperatures and the line profile temperatures. The presence of significant variations between the three measurements in one of the instances discussed demonstrates the value of correlating the information available in many wavelength bands to identify abnormal stars. FUV spectra are also useful for discriminating between DA white dwarfs and other stellar types.

\section{Acknowledgements}

This work was supported by NASA grant NAS5-29298. Many of the spectra used were obtained from the IUE Archives via the National Space Sciences Data Center (NSSDC).

\section{References}

Boggess, A., et al. 1978a, Nature, 275, 372.

Boggess, A., et al. 1978b, Nature, 275, 377.

Bohlin, R., and Holm, A. 1980, NASA IUE Newsletter No. 10, 37.

Bohlin, R. C. 1986, Ap. J., 308, 1001.

Bohlin, R. 1988, in preparation.

Bohlin, R., and Grillmair, C. 1988a, Ap. J. Suppl., Vol. 66, in press.

Bohlin, R., and Grillmair, C. 1988b, in preparation.

Bowyer, S. 1983, Adv. Space Res. 2, 157.

Finley, D. S. 1988, Ph.D. thesis, University of California, Berkeley.

Greenstein, J. L. 1974, Ap. J., 189, L131.

Holberg, J. B., Wesemael, F., and Basile, J. 1986, Ap. J., 306, 624.

Kahn, S. M., Wesemael, F., Liebert, J., Raymond, J. C., Steiner, J. E., and Shipman, H. L. 1984, Ap. J., 278, 255.

Koester, D., and Schönberner, D. 1986, Astron. Astrophys., 154, 125.

Kondo, M., Watanabe, E., Yutain, M., and Noguchi, T. 1982, Pub. Astr. Soc. Japan, 134, 541.

Liebert, J., Wesemael, F., Sion, E. M., and Wegner, G. 1984, Ap. J., 277, 692.

Liebert, James, Fontaine, G., and Wesemael, F. 1987, Memorie della Societa Astronomica Italiana, 58, 17.

Malina, R. F., Bowyer, S., and Basri, G. 1982, Ap. J., 262, 717.

Pye, J. P., and Page, C. G. 1987, Astronomy from Large Databases: Scientific Objectives and Methodological Approaches, Proc. European Southern Observatory No. 28, F. Murtagh and A. Heck, eds., Garching, 447.

Routly, P. M. 1972, Publ. U. S. Naval Obs., Second Series, 20, Part 3.

Vennes, S., Pelletier, C., Fontaine, G., and Wesemael, F. 1987, Proc. IAU Colloquium 95: The Second Conference on Faint Blue Stars, A. G. D. Philip, D. S. Hayes, and J. Liebert, eds., L. Davis Press, Schenectady, NY, p. 665. 